\title{
A NOTE ON SCALE FUNCTIONS AND THE TIME VALUE OF RUIN FOR LÉVY INSURANCE RISK PROCESSES
}

\author{
ENRICO BIFFIS ${ }^{\star}$ AND ANDREAS E. KYPRIANOU ${ }^{\dagger}$
}

\begin{abstract}
We examine discounted penalties at ruin for surplus dynamics driven by a general spectrally negative Lévy process; the natural class of stochastic processes which contains many examples of risk processes which have already been considered in the existing literature. Following from the important contributions of Zhou (2005) we provide an explicit characterization of a generalized version of the Gerber-Shiu function in terms of scale functions, streamlining and extending results available in the literature.
\end{abstract}

KEYWORDS: scale functions, ruin, spectrally negative Lévy processes, Gerber-Shiu function, Laplace transform.

\section{INTRODUCTION}

Originally motivated by the pricing of American claims, Gerber and Shiu $(1997,1998)$ introduced in risk theory a function that jointly penalizes the present value of the time of ruin, the surplus before ruin and the deficit after ruin for Cramér-Lundberg-type processes. This expected discounted penalty, by now known as the Gerber-Shiu function, has been frequently and recursively studied in settings of increasing generality as well as being the named theme of two international workshops in 2006 and 2008. Although far from exhaustive on account of the sheer volume of relevant literature, a list of key papers which pertains to generalizations of the Cramér-Lundberg process includes for example Dickson (1992, 1993); Gerber and Shiu (1997, 1998); Gerber and Landry (1998); Lin and Willmot (1999); Yang and Zhang (2001); Cai and Dickson (2002); Tsai and Willmot (2002); Cai (2004); Garrido and Morales (2006); Morales (2007); Morales and Olivares (2008). The general setting which fits all of these papers is to model the risk process as having stationary and independent increments with no positive jumps. Excluding of course the undesirable case of monotone decreasing paths, the latter class is commonly referred to as spectrally

Date: May 7, 2009.

${ }^{\star}$ Biffis (E.Biffis@imperial.ac.uk) is at Imperial College Business School, Imperial College London, South Kensington Campus, SW7 2AZ United Kingdom. ${ }^{\dagger}$ Kyprianou (A.Kyprianou@bath.ac.uk) is at the Department of Mathematical Sciences, University of Bath, Claverton Down, Bath, BA2 7AY United Kingdom. 
negative Lévy processes. In the current actuarial setting we refer to them as Lévy insurance risk processes.

A common feature of the existing literature is to reduce the analysis of Gerber-Shiu functions to the study of integro-differential equations and/or Volterra equations. In the case of a compound Poisson jump structure the nature of these equations boils down to conditioning on the first jump and considering the recursive nature of the Gerber-Shiu function.

Whilst intuitively appealing, these approaches can be argued to suffer from some limitations as far as dealing with Lévy risk insurance processes. For example, the integro-differential equation can itself only be worked with under the assumption that there is sufficient smoothness in the Gerber-Shiu function, which is a priori a highly non-trivial fact to establish. The associated Volterra equation (see Section 4 for further details) quickly becomes very involved, with different components of the risk process (e.g., bounded and unbounded variation, or continuous and discontinuous paths) requiring separate consideration. Existing calculations show that it is a lengthy procedure to obtain a Volterra equation for the Gerber-Shiu function in the case of infinite activity Lévy insurance risk processes (i.e., countably infinite negative jumps in bounded intervals of time) as this is typically done via compound Poisson approximation. Another difficulty for Volterra/integro-differential equations is that, apart from a handful of exceptions, there is no general theory which offers an identifiable solution, even in the simplest setting of a general compound Poisson jump structure. One such exceptional example would be the classical case of claims whose jump distribution has a Laplace transform which is rational (c.f.: Borovkov, 1976) which includes for example the phase-type distributions.

Zhou (2005) makes an important contribution from the general point of view of Lévy insurance risk processes and introduces the use of so-called scale functions in his analysis of the Gerber-Shiu function. Although his computations are restricted to the case of a compound Poisson jump structure, the analysis still applies verbatim for the case of a general spectrally negative Lévy process.

In this paper we build on Zhou's results and advocate further the virtues of characterizing expected discounted penalties through the use of scale functions and fluctuation theory of Lévy processes when the surplus is driven by a spectrally negative Lévy process. For a modern overview of the theory of scale functions, see for example Bertoin (1996) and Kyprianou (2006). In these two books one also finds further historical references regarding their use in the work of Takács and Zolotarev in the 1960s, and the work of the Kiev school led by Korolyuk (using what they called the method of resolvents) from the 1970s.

As the forthcoming sample computations will hopefully demonstrate, working with scale functions allows one to deal with Lévy insurance risk 
processes, without having to disentangle, for example, a perturbation from a claims component. The approach equally applies to risk processes characterized by components of bounded and unbounded variation, finite and infinite activity as well as by continuous and discontinuous paths, providing in turn solutions that only require inversion of a single Laplace transform.

From the computational point of view, the scale function approach can rely on the vast literature of Laplace transform methods, which has grown extensively over the last few years, and has found numerous applications in option pricing (e.g.: Duffie, Pan and Singleton, 2000; Lee, 2004) and integro-differential equations (e.g.: Kythe and Puri, 2002; Babolian and Shamloo, 2008). Rogers (2000) and Surya (2008) have provided robust methods for numerically computing scale functions. Until recently there were only a handful of explicitly known examples of scale functions (this fact is also reflected by the small variety of concrete examples of the Gerber-Shiu function). Recent work however has produced many completely explicit examples of scale functions (e.g.: Hubalek and Kyprianou, 2008; Patie, 2008; Chaumont, Kyprianou and Pardo, 2009) and in particular Hubalek and Kyprianou (2008); Kyprianou and Rivero (2008); Kyprianou and Patie (2008) outline a method from which many more examples can be computed than the articles themselves had the space for.

Other advantages of scale functions can be seen in recent literature that looks at dividend payments (for example in the form of reflection or refraction strategies) and have proved to be key in understanding optimal barrier strategies (see: Zhou, 2005; Renaud and Zhou, 2007; Kyprianou and Palmowski, 2007; Albrecher, Renaud and Zhou, 2008; Kyprianou and Zhou, 2009; Loeffen, 2009; Kyprianou, Rivero and Song, 2008b; Kyprianou and Loeffen, 2008; Loeffen, 2008a,b). In the context of last ruin times Chiu and Yin (2005) and Baurdoux (2008) also make extensive use of the theory of scale functions. It can be argued that the role and functional robustness of the scale function across this broad range of topics provides a unified reading. Moreover, there is a setting to which the use of more general analogues of scale functions pertains; that is the first passage problem of a general Lévy process and similar such processes such as Markov additive processes and positive selfsimilar processes. Here one also finds a growing body of work (e.g.: Doney and Kyprianou, 2006; Klüppelberg and Erder, 2008; Breuer, 2008; Kyprianou, Pardo and Rivero, 2008a; Chaumont, Kyprianou and Pardo, 2009; Caballero and Chaumont, 2006; Chen and Sheu, 2009).

On the downside, scale functions are arguably a particular phenomenon of risk processes with stationary and independent increments and negative jumps. For example, models of risk which depart from the Poissonian jump structure or introduce path dependencies (and therefore destroy the convenience of stationary independent increments) do 
not necessarily have a comfortable analogue of the theory of scale functions. Moving outside the class of Lévy insurance risk processes however is not completely hopeless as far as scale functions are concerned. For example, the recent paper of Gerber, Lin and Yang (2006) shows that a more general notion of the scale function exists for risk processes which are modelled as strong Markov processes with stationary increments and no positive jumps. Whilst it is not clear exactly how far one may push the theory of scale functions, it is worthy of note that in cases where the use of scale functions fail, the use of Volterra/integrodifferential equations is often still applicable.

The remainder of this note is organized as follows. In Section 2, we look in more detail at the decomposition of a spectrally negative Lévy process in relation to existing models and consider a general class of expected discounted penalty functions (as introduced in Biffis and Morales, 2008) for which we shall exemplify the use of scale functions with some sample computations collected in the form of a main theorem and proof. In Section 3 we give concrete examples making use of recent explicit examples of scale functions. Section 4 offers some concluding remarks.

\section{LÉVy INSURANCE RISK PROCESSES AND DISCOUNTED PENALTIES}

Recall that the Cramér-Lundberg model corresponds to a Lévy process $X=\left\{X_{t}: t \geq 0\right\}$ with law $\mathbb{P}$ and characteristic exponent given by

$$
\Psi(\theta)=-\log \int_{\mathbb{R}} e^{i \theta x} \mathbb{P}\left(X_{1} \in d x\right)=-i c \theta+\lambda \int_{(0, \infty)}\left(1-e^{-i \theta x}\right) F(d x),
$$

for $\theta \in \mathbb{R}$ such that $\lim _{t \uparrow \infty} X_{t}=\infty$. In other words, $X$ is a compound Poisson process with arrival rate $\lambda>0$ and negative jumps, corresponding to claims, having common distribution function $F$ with finite mean $1 / \mu$ as well as a drift $c>0$, corresponding to a steady income due to premiums, which necessarily satisfies $c-\lambda / \mu>0$; the safety loading condition.

As mentioned in the introduction, we work with a general spectrally negative Lévy process $X=\left\{X_{t}: t \geq 0\right\}$ and refer to it as a Lévy insurance risk process. The analogous condition to the safety loading condition in this general setting (that is to say, the necessary and sufficient condition that ensures $\left.\lim _{t \uparrow \infty} X_{t}=\infty\right)$ is $\mathbb{E}\left(X_{1}\right) \in(0, \infty)$. (Note that it is impossible for the latter expectation to equal $+\infty$, cf. Chapter VII of Bertoin, 1996, or Chapter 8 of Kyprianou, 2006). Under the latter assumption the Lévy-Khintchine formula for the characteristic exponent of a Lévy insurance risk process may always be written in 
the form

$$
\begin{aligned}
\Psi(\theta) & =-\log \int_{\mathbb{R}} e^{i \theta x} \mathbb{P}\left(X_{1} \in d x\right) \\
& =-i \theta a+\frac{1}{2} \sigma^{2} \theta^{2}+\int_{(0, \infty)}\left(1-e^{-i \theta x}-i \theta x\right) \Pi(d x)
\end{aligned}
$$

for $\theta \in \mathbb{R}$, where $a>0, \sigma \geq 0$, the Lévy measure, $\Pi$, is concentrated on $(0, \infty)$ and necessarily satisfies $\int_{(0, \infty)}\left(x \wedge x^{2}\right) \Pi(d x)<\infty$. Note that, although jumps are negative, we have made the unusual arrangement in the Lévy-Khintchine formula of supporting the Lévy measure $\Pi$ on the positive half line. Moreover, not using a truncation function against the linear term in the integrand of $\Psi$, restricting $a>0$ and allowing integrability against $\Pi$ of $\left(x \wedge x^{2}\right)$ as opposed to the usual $\left(1 \wedge x^{2}\right)$ are all adjustments to the usual form of the Lévy-Khintchine formula which are possible thanks to the safety loading condition $\mathbb{E}\left(X_{1}\right) \in(0, \infty)$.

Different authors interpret the different parts of the characteristic exponent, and the associated Lévy-Itô decomposition, in different ways. The case that $\Pi(0, \infty)<\infty$ corresponds to compound Poisson jumps with arrival rate $\lambda=\Pi(0, \infty)$ and jump distribution $F(d x)=\Pi(d x) / \Pi(0, \infty)$. In particular when, in addition, $\sigma=0$, this is the classical Cramér-Lundberg model with $c=a+\int_{(0, \infty)} x \Pi(d x)$. The case that $\Pi(0, \infty)=\infty$ but $\int_{(0,1)} x \Pi(d x)<\infty$ is the case of a bounded variation jump process and therefore $X$ is necessarily the difference of a linear drift, also with rate $a+\int_{(0, \infty)} x \Pi(d x)$, and an infinite activity subordinator plus an independent Brownian motion with volatility $\sigma$. This model was the subject of the recent work of, for example, Morales (2007). The case that $\Pi(0, \infty)=\infty$ but $\int_{(0,1)} x \Pi(d x)=\infty$ is the case of an unbounded variation jump part. When $\sigma=0$, this latter case includes, for example, the model of Furrer (1998) involving stable processes of index $\alpha \in(1,2)$.

Many authors (including the aforementioned) also prefer to talk about perturbation of the classical Cramér-Lundberg process by another Lévy process, a typical and general case at hand being found in the work of Huzak, Perman, Šikić and Vondraček (2004) and Morales and Olivares (2008). Since the sum of two independent Lévy processes is again a Lévy process, the concept of perturbation is contained in the above setting by decomposing $\Pi=\Pi^{(1)}+\Pi^{(2)}$, where $\Pi^{(1)}$ corresponds to the Lévy measure of the Cramér-Lundberg process and $\Pi^{(2)}$ is that of the perturbation process. If the perturbation is a Brownian motion, then this simply corresponds to the case $\sigma \neq 0$.

The generalized version of the Gerber-Shiu function that we shall be interested in here for the purpose of exemplifying the mathematical robustness of scale functions, was recently presented in Biffis and Morales (2008). It includes information on the distribution of the last minimum 
of the surplus before ruin, $\underline{X}_{\tau-}:=\inf _{t<\tau} X_{t}$, with $\tau:=\inf \left\{t>0: X_{t}<\right.$ 0 ) denoting the ruin time of $X$.

Definition 1. Let $f: \mathbb{R}^{3} \rightarrow[0, \infty)$ be a bounded measurable function such that $f(0, \cdot, \cdot)=0$ and $x, q \geq 0$. For a Lévy insurance risk process, $X$, starting at $x$ (whose law and expectation operator we shall denote by $\mathbb{P}_{x}$ and $\mathbb{E}_{x}$ respectively), the discounted penalty function associated with $f$ and $q$ is given by

$$
\phi_{f}(x, q)=\mathbb{E}_{x}\left(e^{-q \tau} f\left(-X_{\tau}, X_{\tau-}, \underline{X}_{\tau-}\right) \mathbf{1}_{\{\tau<\infty\}}\right)
$$

Our method for handling the general form of $\phi_{f}(x, q)$ given above revolves around the simple fact that any such function may always be written as an integral of $f$ against a (possibly defective) probability kernel. The latter being the time-discounted triple law coding the overshoot-undershoot distribution of $\left(-X_{\tau}, X_{\tau-}, \underline{X}_{\tau-}\right)$. Our main theorem, below, provides an exact analytical characterization of this kernel in terms of scale functions which we now formally introduce.

Firstly let

$$
\psi(\theta)=-\Psi(-i \theta)=\log \mathbb{E}\left(e^{\theta X_{1}}\right)
$$

be the Laplace exponent of $X$ which is known to be finite for at least $\theta \in$ $[0, \infty)$. The asymptotic behaviour of $X$ is characterized by $\psi^{\prime}(0+)$, so that $X$ drifts to $\pm \infty$ (oscillates) accordingly as $\pm \psi^{\prime}(0+)>0\left(\psi^{\prime}(0+)=\right.$ $0)$.

For every $q \geq 0$ there exists a function $W^{(q)}: \mathbb{R} \rightarrow[0, \infty)$ such that $W^{(q)}(x)=0$ for all $x<0$ and otherwise is absolutely continuous on $(0, \infty)$ satisfying,

$$
\int_{0}^{\infty} e^{-\lambda x} W^{(q)}(x) d x=\frac{1}{\psi(\lambda)-q}, \quad \text { for } \quad \lambda>\Phi(q),
$$

where $\Phi(q)$ is the largest solution to the equation $\psi(\theta)=q$ (there are at most two solutions). For short we shall write $W^{(0)}=W$. When $X$ has paths of unbounded variation then it is known that $W^{(q)}$ is in fact continuously differentiable on $(0, \infty)$. In particular, when $X$ has a Gaussian component then $W^{(q)}$ is twice continuously differentiable on $(0, \infty)$; cf. Chan et al. (2009). Otherwise, when $X$ has paths of bounded variation, a necessary and sufficient condition for $W^{(q)}$ to be continuous is that the jump measure $\Pi$ has no atoms (cf. Lambert, 2000; Kyprianou, Rivero and Song, 2008b). However, the continuous differentiability of $W^{(q)}$ is used nowhere in the statement or proof of our main result below.

The following theorem characterizes the discounted penalty (2) in terms of scale functions of Lévy insurance risk processes. 
Theorem 1. Suppose that $X$ is a Lévy insurance risk process. The expected discounted penalty function defined in (2) is then given by

$$
\phi_{f}(x, q)=\int_{(0, \infty)^{3}} \mathbf{1}_{\{v \geq y\}} f(u, v, y) K_{x}^{(q)}(d u, d v, d y)
$$

where

$$
\begin{aligned}
& K_{x}^{(q)}(d u, d v, d y) \\
& \quad=e^{-\Phi(q)(v-y)}\left\{W^{(q) \prime}(x-y)-\Phi(q) W^{(q)}(x-y)\right\} \Pi(d u+v) d y d v .
\end{aligned}
$$

Remark 1. Insisting that $f(0, \cdot, \cdot)=0$ simply means that the function $\phi_{f}(x, q)$ concerns itself only with the joint law of the overshoot and undershoots. It is known from the general phenomenon of creeping of Lévy processes that $\underline{X}_{\tau-}=X_{\tau-}=0$ on $\left\{X_{\tau}=0\right\}$ and this occurs with probability

$$
\mathbb{E}_{x}\left(e^{-q \tau} ; X_{\tau}=0\right)=\frac{\sigma^{2}}{2}\left\{W^{(q) \prime}(x)-\Phi(q) W^{(q)}(x)\right\}
$$

(cf. Pistorius, 2005). Note that we take advantage of the fact that the quantity $W^{(q) \prime}$ is well defined for all $x \geq 0$ when $\sigma \neq 0$ (according to remarks earlier concerning smoothness properties of $\left.W^{(q)}\right)$. The possibility of creeping accounts for the fact that $K_{x}^{(q)}$ is defective when $\sigma \neq 0$.

Proof of Theorem 1. Throughout this proof we shall make frequent reference to what, by now, may be considered to be standard facts concerning scale functions. The reader is directed to Chapter 8 of Kyprianou (2006) for further background reading.

We prove the result for the case that $q>0$. The result for the case $q=0$ follows simply by taking limits as $q \downarrow 0$, taking account of the fact that $\Phi(0)>0$ if and only if $\mathbb{E}\left(X_{1}\right)<0$. It will be convenient to introduce the spectrally positive Lévy process $Y=-X$. Then the problem is to compute

$$
\mathbb{E}\left(e^{-q \sigma_{x}} f\left(Y_{\sigma_{x}}-x, x-Y_{\sigma_{x}-}, x-\bar{Y}_{\sigma_{x}-}\right) \mathbf{1}_{\left\{\sigma_{x}<\infty\right\}}\right)
$$

or equivalently

$$
\mathbb{E}\left(e^{-q \sigma_{x}} ; Y_{\sigma_{x}}-x \in d u, x-Y_{\sigma_{x}-} \in d v, x-\bar{Y}_{\sigma_{x}-} \in d y\right),
$$

where $\sigma_{x}:=\inf \left\{t>0: Y_{t}>x\right\}, \bar{Y}_{\sigma_{x}-}:=\sup _{t<\sigma_{x}} Y_{t}$, and in the second expectation, for convenience we implicitly understand $\sigma_{x}<\infty$.

According to the quintuple law (see Example 8 in Doney and Kyprianou, 2006), in the case that $X$ drifts to $\infty$ (and hence $\Phi(0)=0$ ), we have for $u, v>0$ and $0<y \leq v \wedge x$,

$$
\begin{aligned}
\mathbb{P}\left(Y_{\sigma_{x}}-x \in d u, x-Y_{\sigma_{x}-}\right. & \left.\in d v, x-\bar{Y}_{\sigma_{x}-} \in d y\right) \\
& =k W(x-d y) \Pi(d u+v) d v \\
& =k W^{\prime}(x-y) \Pi(d u+v) d y d v
\end{aligned}
$$


where $W$ is the 0 -scale function associated to $X$ having Laplace transform $1 / \psi(\lambda), W^{\prime}$ is a version of its density, and $k$ is a constant which depends on the normalization of the local time of $Y$ at its supremum.

We claim that the constant $k$ is unity. Indeed note that, on the one hand, (4) tells us that

$$
\mathbb{P}\left(Y_{\sigma_{x}} \neq x\right)=k \int_{0}^{x} d y \cdot W^{\prime}(x-y) \int_{0}^{\infty} \bar{\Pi}(z+y) d z .
$$

On the other hand, appealing to the method of resolvents described at the end of Section 8.4 of Kyprianou (2006) and recalling that $X$ drifts to $\infty$ (and hence $\Phi(0)=0$ ), we also know that

$$
\mathbb{P}_{x}\left(-X_{\tau} \in d u, X_{\tau-} \in d v\right)=\Pi(d u+v)\{W(x)-W(x-v)\} d v
$$

for $u, v>0$. Integrating out $u, v>0$ we obtain

$$
\begin{aligned}
\mathbb{P}\left(Y_{\sigma_{x}} \neq x\right) & =\int_{0}^{\infty} \bar{\Pi}(v)\{W(x)-W(x-v)\} d v \\
& =\int_{0}^{\infty} \bar{\Pi}(v) \int_{0}^{v} W^{\prime}(x-y) d y d v \\
& =\int_{0}^{x} d y \cdot W^{\prime}(x-y) \int_{y}^{\infty} \bar{\Pi}(v) d v \\
& =\int_{0}^{x} d y \cdot W^{\prime}(x-y) \int_{0}^{\infty} \bar{\Pi}(z+y) d z .
\end{aligned}
$$

Comparing the two equations for $\mathbb{P}\left(Y_{\sigma_{x}} \neq x\right)$ we are led to the conclusion that $k=1$, thus justifying the claim.

To complete the proof, we need to develop the expression (4) so that it incorporates exponential discounting. However, this can be done as a simple consequence of applying the exponential change of measure

$$
\left.\frac{d \mathbb{P}^{\Phi(q)}}{d \mathbb{P}}\right|_{\mathcal{F}_{t}}=e^{\Phi(q) X_{t}-q t}
$$

where $\Phi(q)=\sup \{\lambda \geq 0: \psi(\lambda)=q\}$ and $\mathcal{F}_{t}=\sigma\left(X_{s}: s \leq t\right)$. Recall that under $\mathbb{P}^{\Phi(q)}$ the process $X$ is still spectrally negative, and, irrespective of the value of $\mathbb{E}\left(X_{1}\right)$, still drifts to $\infty$. Moreover, the 0 scale function of $X$ under $\mathbb{P}^{\Phi(q)}$, which we write as $W_{\Phi(q)}(x)$, is related to the $q$-scale function of $X$ under $\mathbb{P}$, that is to say $W^{(q)}(x)$, via the relation

$$
W^{(q)}(x)=e^{\Phi(q) x} W_{\Phi(q)}(x) .
$$

Making use of (4) but under the law $\mathbb{P}^{\Phi(q)}$ instead, we may now write

$$
\begin{aligned}
& \mathbb{E}\left(e^{-q \sigma_{x}} ; Y_{\sigma_{x}}-x \in d u, x-Y_{\sigma_{x}-} \in d v, x-\bar{Y}_{\sigma_{x}-} \in d y\right) \\
& =e^{\Phi(q)(x+u)} \mathbb{P}^{\Phi(q)}\left(Y_{\sigma_{x}}-x \in d u, x-Y_{\sigma_{x}-} \in d v, x-\bar{Y}_{\sigma_{x}-} \in d y\right) \\
& =e^{\Phi(q)(x+u)} W_{\Phi(q)}(x-d y) \Pi_{\Phi(q)}(d u+v) d v \\
& =e^{\Phi(q)(x+u)} W_{\Phi(q)}^{\prime}(x-y) \Pi_{\Phi(q)}(d u+v) d y d v
\end{aligned}
$$


where $\Pi_{\Phi(q)}$ is the jump measure associated with $\left(X, \mathbb{P}^{\Phi(q)}\right)$ and $W_{\Phi(q)}^{\prime}$ is a version of the density of $W_{\Phi(q)}$. It is known that $\Pi_{\Phi(q)}(d x)=$ $e^{-\Phi(x) x} \Pi(d x)$. Plugging this in we get

$$
\begin{aligned}
& \mathbb{E}\left(e^{-q \sigma_{x}} ; Y_{\sigma_{x}}-x \in d u, x-Y_{\sigma_{x}-} \in d v, x-\bar{Y}_{\sigma_{x}-} \in d y\right) \\
& =e^{\Phi(q)(x+u)} W_{\Phi(q)}^{\prime}(x-y) e^{-\Phi(q)(u+v)} \Pi(d u+v) d y d v \\
& =e^{-\Phi(q)(v-y)} e^{\Phi(q)(x-y)} W_{\Phi(q)}^{\prime}(x-y) \Pi(d u+v) d y d v .
\end{aligned}
$$

From the information given earlier on scale functions, we may differentiate $W_{\Phi(q)}$ almost everywhere. It follows that we may differentiate (6) almost everywhere resulting in a density which satisfies

$$
W^{(q) \prime}(x)-\Phi(q) W^{(q)}(x)=e^{\Phi(q) x} W_{\Phi(q)}^{\prime}(x) .
$$

Taking advantage of the above formula we deduce that

$$
\begin{aligned}
& \mathbb{E}\left(e^{-q \sigma_{x}} ; Y_{\sigma_{x}}-x \in d u, x-Y_{\sigma_{x}-} \in d v, x-\bar{Y}_{\sigma_{x}-} \in d y\right) \\
& =e^{-\Phi(q)(v-y)}\left\{W^{(q) \prime}(x-y)-\Phi(q) W^{(q)}(x-y)\right\} \Pi(d u+v) d y d v,
\end{aligned}
$$

where $u, v>0,0<y \leq v \wedge x$. Note in particular when $q=0$, recalling that $\Phi(0)=0$ as the process drift to $-\infty$, we see agreement with the formula (4) and the proof is complete.

Remark 2. As alluded to in the introduction, the identity (5) was found in Zhou (2005) for the case of a compound Poisson jump structure, and the method we have appealed here to obtain (5) is a variant of the technique used by Zhou.

\section{EXAmples}

It was indicated in the introduction that, until recently, a strong criticism of the use of scale functions is that there has been little progress in producing real concrete examples over and above the core classical cases of the Cramér-Lundberg model with (mixed) exponential jumps and a Brownian perturbation or the spectrally negative stable model. We also indicated in the same paragraph that recent process has been made in this direction, citing a small cluster of papers dated no earlier than 2008. To give strength to what this progress in the theory of scale functions means in this context, we shall offer a number of explicit examples below.

Example 1. Suppose that $X$ is a spectrally negative tempered stable process and therefore has Laplace exponent of the form

$$
\psi(\theta)=(\theta+c)^{\alpha}-c^{\alpha},
$$

where $\theta \geq 0, c \geq 0$ and $\alpha \in(1,2)$. Note that $\mathbb{E}\left(X_{1}\right)=\psi^{\prime}(0+)=$ $\alpha c^{\alpha-1} \geq 0$ and so the process drifts to infinity if and only if $c>0$ and otherwise, when $c=0$, it oscillates. The latter case corresponds to an $\alpha$-stable process which was studied by Furrer (1998). From the analytical form of the Laplace exponent one sees that the law of $X$ 
is the result of an exponential change of measure applied to the law of an $\alpha$-stable process with stability index $\alpha$. In this respect it is straightforward to deduce that $X$ has no Gaussian component, is a process of unbounded variation and its Lévy measure is given by

$$
\Pi(d x)=\frac{1}{\Gamma(-\alpha)} \frac{e^{-c x}}{x^{1+\alpha}} d x, x>0 .
$$

Here $\Gamma(u)$ is the usual gamma function which is defined on $\mathbb{R}$ with the exception of the points $\{0,-1,-2, \ldots\}$. It is also an easy exercise to show that $\Phi(q)=\left(q+c^{\alpha}\right)^{1 / \alpha}-c$. In Kyprianou and Patie (2008) it was shown that

$$
W^{(q)}(x)=e^{-c x} x^{\alpha-1} \mathcal{E}_{\alpha, \alpha}\left(\left(q+c^{\alpha}\right) x^{\alpha}\right),
$$

where

$$
\mathcal{E}_{\alpha, \beta}(x)=\sum_{n=0}^{\infty} \frac{x^{n}}{\Gamma(\alpha n+\beta)}
$$

is the two-parameter Mittag-Leffler function.

Next recalling that $W_{\Phi(q)}(x)=e^{-\Phi(q) x} W^{(q)}(x)$ we may note from $(7)$ that for $u, v, y>0$ and $y \leq v$,

$$
\begin{aligned}
& \mathbb{E}_{x}\left(e^{-q \tau} ;-X_{\tau} \in d u, X_{\tau-} \in d v, \underline{X}_{\tau-} \leq y\right) \\
& =\frac{1}{\Gamma(-\alpha)} e^{\left(\left(q+c^{\alpha}\right)^{1 / \alpha}-c\right)(x-v)} \frac{e^{-c(u+v)}}{(u+v)^{1+\alpha}} \\
& \times\left\{e^{-\left(q+c^{\alpha}\right)^{1 / \alpha} x} x^{\alpha-1} \mathcal{E}_{\alpha, \alpha}\left(\left(q+c^{\alpha}\right) x^{\alpha}\right)\right. \\
& \left.\quad \quad-e^{-\left(q+c^{\alpha}\right)^{1 / \alpha}(x-y)}(x-y)^{\alpha-1} \mathcal{E}_{\alpha, \alpha}\left(\left(q+c^{\alpha}\right)(x-y)^{\alpha}\right)\right\} d u d v
\end{aligned}
$$

which, although somewhat ugly, is sufficiently explicit to work with in the context of a programme such as MATLAB or Mathematica.

The situation becomes somewhat more amicable when $q=0$ and one is only concerned with the overshoot and undershoots as the next examples illustrate.

Example 2. Consider the case that $X$ is a spectrally negative Lévy process with Laplace exponent given by

$$
\psi(\theta)=\kappa \theta+c \Gamma(-\beta) \theta\left(\gamma^{\beta}-(\gamma+\theta)^{\beta}\right),
$$

where $\theta, \kappa \geq 0$ and $\beta \in(0,1)$. It was shown in Hubalek and Kyprianou (2008) that the process $X$ has no Gaussian component, has paths of unbounded variation, and its Lévy measure is given by

$$
\Pi(d x)=c e^{-\gamma x}\left(\frac{\gamma x+(\beta+1)}{x^{\beta+2}}\right) d x .
$$

We may easily check that $\psi^{\prime}(0+)=\kappa$ showing that the process $X$ drifts to infinity if and only if $\kappa>0$ and otherwise, when $\kappa=0$, it oscillates. 
It was also shown in the aforementioned paper that

$$
W(x)=-\frac{1}{c \Gamma(-\beta)} \int_{0}^{x} e^{-\gamma y} y^{\beta-1} \mathcal{E}_{\beta, \beta}\left(\frac{\kappa+c \Gamma(-\beta) \gamma^{\beta} y^{\beta}}{c \Gamma(-\beta)}\right) d y .
$$

Appealing to (4) we see that for $u, v, y>0$ and $y \leq v$,

$$
\begin{aligned}
\mathbb{P}_{x}\left(-X_{\tau} \in d u, X_{\tau-} \in d v, \underline{X}_{\tau-} \in d y\right) \\
=-\frac{1}{\Gamma(-\beta)} e^{-\gamma(x-y+u+v)}\left(\frac{\gamma(u+v)+(\beta+1)}{(u+v)^{\beta+2}}\right) \\
\quad \times(x-y)^{\beta-1} \mathcal{E}_{\beta, \beta}\left(\frac{\kappa+c \Gamma(-\beta) \gamma^{\beta}(x-y)^{\beta}}{c \Gamma(-\beta)}\right) d u d y d v
\end{aligned}
$$

which is again sufficiently explicit to work with numerically if not analytically.

Example 3. Now consider the spectrally negative Lévy process with Laplace exponent

$$
\psi(\theta)=\frac{\theta \Gamma(\nu+\beta \theta+\lambda)}{c \beta \Gamma(\nu+\beta \theta)},
$$

where $\theta \geq 0, \beta, c>0, \nu \geq 0$ and $\lambda \in(0,1)$. In Kyprianou and Rivero (2008) it was shown that the underlying Lévy process has no Gaussian component and its Lévy measure satisfies

$$
\Pi(x, \infty)=\frac{\lambda}{c \beta^{2} \Gamma(1-\lambda)} \frac{e^{x(1-\nu) / \beta}}{\left(e^{x / \beta}-1\right)^{\lambda+1}} .
$$

A straightforward calculation shows that

$$
\psi^{\prime}(0+)=\frac{\Gamma(\nu+\lambda)}{c \beta \Gamma(\nu)} \geq 0
$$

which implies that $X$ drifts to infinity if and only if $\nu>0$ and otherwise it oscillates. In the same paper it was also shown that

$$
W(x)=c \int_{0}^{x} \frac{e^{-z(\nu+\lambda-1) / \beta}}{\Gamma(\lambda)}\left(e^{z / \beta}-1\right)^{\lambda-1} d z .
$$

In that case

$$
\begin{aligned}
\mathbb{P}_{x}( & \left.-X_{\tau}>u, X_{\tau-} \in d v, \underline{X}_{\tau-} \in d y\right) \\
& =\frac{\lambda}{\beta^{2} \Gamma(1-\lambda) \Gamma(\lambda)} \frac{e^{-(x-y) \nu / \beta}\left(1-e^{-(x-y) / \beta}\right)^{\lambda-1} e^{(u+v)(1-\nu) / \beta}}{\left(e^{(u+v) / \beta}-1\right)^{\lambda+1}} d y d v .
\end{aligned}
$$

Again this is a formula which has a degree of analytical simplicity and otherwise is suitable for numerical use.

We close this section by reiterating that these three examples are by no means special. Hubalek and Kyprianou (2008); Kyprianou and Rivero (2008); Kyprianou and Patie (2008) contain many more examples as well as methodologies for generating a plethora of other explicit examples; that is to say, examples for which the scale function and the characteristic triplet of the underlying Lévy process may be expressed 
in full analytic detail. At the same time, it is fair to say that these articles offer a lot more in terms of scale functions with $q=0$ than the case $q>0$. In this respect there is still more that can be done with regard to producing explicit examples of scale functions when $q>0$.

\section{Conclusion}

In this short communication, our aim has been to provide a convincing case for the re-alignment of the classical ruin problem with the modern theory of scale functions for spectrally negative Lévy processes. We have provided an explicit expression for a (generalized) version of the Gerber-Shiu function which umbrellas all previously considered cases of risk processes with stationary and independent increments and no positive jumps. From the point of view of numerical or detailed analytical examples, the Gerber-Shiu function requires the inversion of one Laplace transform and, in both cases, a solid base of literature already exists for further exploitation. We feel that it is also important to note that the benefits of scale functions are not only to be seen when moving out of the class of Cramér-Lundberg models into more exotic examples of Lévy insurance risk processes. As Zhou (2005) himself illustrates together with the case we have made here, in the classical setting alone, all of the virtues of scale functions are still of pertinent value.

Finally, let us close with some remarks on the relationship between scale functions, integro-differential equations and Volterra equations as this is not necessarily as clear as one might hope for. The following toy example gives some insight. Firstly we recall the, by now, classical two sided exit formula (cf. Chapter 8 of Kyprianou, 2006)

$$
\mathbb{E}_{x}\left(e^{-q T_{a}} \mathbf{1}_{\left\{T_{a}<\tau\right\}}\right)=\frac{W^{(q)}(x)}{W^{(q)}(a)},
$$

where $x, q \geq 0, a>0$ and $T_{a}=\inf \left\{t>0: X_{t}>a\right\}$. Making use of the fact that $X_{T_{a}}=a$ on $\left\{T_{a}<\infty\right\}, W^{(q)}(x)=0$ for $x<0$ and $W^{(q)}(0)=0$ when $X$ creeps below zero, one notes that $W^{(q)}\left(X_{T_{a} \wedge \tau}\right) / W^{(q)}(a)=\mathbf{1}_{\left\{T_{a}<\tau\right\}}$. A well known argument which follows from the latter observation and an application of the strong Markov property, tells us that

$$
\begin{aligned}
\mathbb{E}_{x}\left(e^{-q T_{a}} \mathbf{1}_{\left\{T_{a}<\tau\right\}} \mid \mathcal{F}_{t}\right) & =\mathbb{E}_{x}\left(e^{-q\left(T_{a} \wedge \tau\right)} \frac{W^{(q)}\left(X_{T_{a} \wedge \tau}\right)}{W^{(q)}(a)} \mid \mathcal{F}_{t}\right) \\
& =\left.e^{-q\left(t \wedge T_{a} \wedge \tau\right)} \mathbb{E}_{z}\left(e^{-q\left(T_{a} \wedge \tau\right)} \frac{W^{(q)}\left(X_{\left.T_{a} \wedge \tau\right)}\right)}{W^{(q)}(a)}\right)\right|_{z=X_{t \wedge T_{a} \wedge \tau}} \\
& =\left.e^{-q\left(t \wedge T_{a} \wedge \tau\right)} \mathbb{E}_{z}\left(e^{-q T_{a}} \mathbf{1}_{\left\{T_{a}<\tau\right\}}\right)\right|_{z=X_{t \wedge T_{a} \wedge \tau}} \\
& =e^{-q\left(t \wedge T_{a} \wedge \tau\right)} \frac{W^{(q)}\left(X_{t \wedge T_{a} \wedge \tau}\right)}{W^{(q)}(a)}
\end{aligned}
$$


where $\mathcal{F}_{t}$ is the natural filtration generated by $\left\{X_{s}: s \leq t\right\}$ satisfying the usual conditions and the last equality follows from (8). This shows that the quantity on the right hand side above is a martingale and in particular, assuming that $W^{(q)}$ is sufficiently smooth, by considering its semi-martingale representation, it must follow that $(\Gamma-q) W^{(q)}(x)=0$ for $x \in(0, \infty)$, where $\Gamma$ is the infinitesimal generator of $X$. The line of reasoning which leads to the latter is standard, however, we give a brief sketch proof here none the less. Let us assume for convenience that $X$ has a compound Poisson jump structure, then a straightforward application of Itô's formula, taking note of the Lévy-Itô decomposition and that $W^{(q)}$ is twice continuously differentiable on $(0, \infty)$, implies that

$$
\begin{aligned}
& e^{-q\left(t \wedge T_{a} \wedge \tau\right)} W^{(q)}\left(X_{t}\right)-W^{(q)}(x) \\
& \quad=-q \int_{0}^{t \wedge T_{a} \wedge \tau} e^{-q s} W^{(q)}\left(X_{s}\right) d s+\frac{1}{2} \sigma^{2} \int_{0}^{t \wedge T_{a} \wedge \tau} e^{-q s} W^{(q) \prime \prime}\left(X_{s}\right) d s \\
& \quad+c \int_{0}^{t \wedge T_{a} \wedge \tau} e^{-q s} W^{(q) \prime}\left(X_{s}\right) d s+\sigma \int_{0}^{t \wedge T_{a} \wedge \tau} e^{-q s} W^{(q) \prime}\left(X_{s}\right) d B_{s} \\
& \quad+\int_{0}^{t \wedge T_{a} \wedge \tau} \int_{(0, \infty)}\left\{f\left(X_{s-}-y\right)-f\left(X_{s-}\right)\right\} \Pi(d y) d s \\
& +M_{t}
\end{aligned}
$$

where $B=\left\{B_{t}: t \geq 0\right\}$ is the Brownian component with coefficient $\sigma \geq 0, c$ is a real-valued constant which must satisfy $c>0$ if $\sigma=0$ (which avoids the possibility that $X$ has monotone paths) and

$$
\begin{aligned}
M_{t}= & \sum_{s \leq t \wedge T_{a} \wedge \tau}\left\{f\left(X_{s}\right)-f\left(X_{s-}\right)\right\} \\
& -\int_{0}^{t \wedge T_{a} \wedge \tau} \int_{(0, \infty)}\left\{f\left(X_{s-}-y\right)-f\left(X_{s-}\right)\right\} \Pi(d y) d s .
\end{aligned}
$$

Recall that for $x>0$

$$
\begin{aligned}
(\Gamma-q) W^{(q)}(x) \\
\quad=\frac{1}{2} \sigma^{2} W^{(q) \prime \prime}(x)+c W^{(q) \prime}(x) \\
\quad+\int_{0}^{\infty}\left\{W^{(q)}(x-y)-W^{(q)}(x)\right\} \Pi(d y)-q W^{(q)}(x) .
\end{aligned}
$$

Noting that the stochastic integral with respect to $B$ and the process $M$ are local martingales, as is the left-hand side of (10), it follows that the bounded variation terms in (10), which are equal to

$$
\int_{0}^{t \wedge T_{a} \wedge \tau}(\Gamma-q) W^{(q)}\left(X_{s}\right) d s
$$

must be equal to zero. This implies that $(\Gamma-q) W^{(q)}(x)=0$ for $x \in$ $(0, a)$ and since we may choose $a>0$ arbitrarily, we have, as claimed, 
that

$$
(\Gamma-q) W^{(q)}(x)=0 \text { for } x \in(0, \infty) .
$$

The above line of reasoning, although becoming more technical, also works for a general spectrally negative Lévy process.

From (12) one may then proceed to convert this integro-differential equation into a Volterra equation by simply integrating the generator equation twice in $x$. A perfect example of how this is carried out can be seen in full detail in the paper of Yin and Wang (2008). Indeed, suppose further to the assumptions made above that the Gaussian coefficient is $\sigma>0$. In that case it can easily be derived from the definition (3) that $W^{(q)}(0+)=0$ and $W^{(q) \prime}(0+)=2 / \sigma^{2}$ (see for example Chan, Kyprianou and Savov, 2009). Setting the right-hand side of (11) equal to zero and integrating twice (this requires judicious integration by parts), the generator equation (12) reduces to the Volterra equation

for $x>0$, where

$$
W^{(q)}(x)+W^{(q)} * g(x)=\frac{2}{\sigma^{2}} x
$$

$$
g(x)=\frac{2}{\sigma^{2}}\left(\int_{0}^{x} \Pi(0, y) d y+c-(q+\Pi(0, \infty)) x\right) .
$$

To complete the circle of computations, one may also take Laplace transforms of the above Volterra equation and deduce that for $\beta$ sufficiently large

$$
\begin{aligned}
\frac{2}{\sigma^{2} \beta^{2}}= & \left(\int_{0}^{\infty} e^{-\beta x} W^{(q)}(x) d x\right) \\
& \times\left[1+\frac{2}{\sigma^{2}}\left(\frac{1}{\beta^{2}} \int_{0}^{\infty} e^{-\beta x} \Pi(d x)+\frac{c}{\beta}-(q+\Pi(0, \infty)) \frac{1}{\beta^{2}}\right)\right],
\end{aligned}
$$

which is equivalent to (3) when one takes account of the fact that

$$
\psi(\beta)=\frac{1}{2} \sigma^{2} \beta^{2}+c \beta-\int_{0}^{\infty}\left(1-e^{-\beta x}\right) \Pi(d x), \quad \beta \geq 0 .
$$

\section{ACKnowledgments}

We are grateful to Hansjörg Albrecher, Ronnie Loeffen, Xiaowen Zhou and two anonymous referees for their helpful comments on, and meticulous reading of, earlier versions of this paper.

\section{REFERENCES}

Albrecher, H., J.-F. Renaud and X. Zhou (2008). A Lévy insurance risk process with tax. J. Appl. Probab., vol. 45(2):363-375.

Babolian, E. and A. Shamloo (2008). Numerical solution of Volterra and integro-differential equations of convolution type by using operational matrices of piecewise constant orthogonal functions. J. Comp. Appl. Math., vol. 214(2):495-508. 
Baurdoux, E. (2008). Last exit before an exponential time for spectrally negative Lévy processes. To appear in Adv. Appl. Probab..

Bertoin, J. (1996). Lévy Processes. Cambridge Tracts in Mathematics 121. Cambridge University Press.

Biffis, E. and M. Morales (2008). On a generalization of the GerberShiu function to path-dependent penalties. Tech. rep.

Borovkov, A. (1976). Stochastic processes in queueing theory. Applications of Mathematics No. 4. Springer-Verlag.

Breuer, L. (2008). A quintuple law for Markov-additive processes with phase-type jumps. To appear in J. Appl. Probab..

Caballero, M. E. and L. Chaumont (2006). Conditioned stable Lévy processes and the Lamperti representation. J. Appl. Probab., vol. 43(4):967-983.

Cai, J. (2004). Ruin probabilities and penalty functions with stochastic rates of interest. Stoch. Proc. Appl., vol. 112(1):53-78.

Cai, J. and D. C. M. Dickson (2002). On the expected discounted penalty function at ruin of a surplus process with interest. Insurance Math. Econom., vol. 30(3):389-404.

Chan, T., A. E. Kyprianou and M. Savov (2009). Smoothness of scale functions for spectrally negative Lévy processes. Tech. rep., http://arxiv.org/abs/0903.1467.

Chaumont, C., A. Kyprianou and J. Pardo (2009). Some explicit identities associated with positive self-similar Markov processes. Stoch. Proc.. Appl., vol. 119(3):980-1000.

Chen, Y.-T. and Y.-C. Sheu (2009). A note on $r$-balayages of matrixexponential Lévy processes. Electron. Comm. Probab., vol. 14:165-175.

Chiu, S. N. and C. Yin (2005). Passage times for a spectrally negative Lévy process with applications to risk theory. Bernoulli, vol. 11(3):511522 .

Dickson, D. C. M. (1992). On the distribution of the surplus prior to ruin. Ins. Math. Econ., vol. 11(3):191-207.

Dickson, D. C. M. (1993). On the distribution of the claim causing ruin. Insurance Math. Econom., vol. 12(2):143-154.

Doney, R. and A. Kyprianou (2006). Overshoots and undershoots of Lévy processes. Ann. Appl. Probab., vol. 16(1):91-106.

Duffie, D., J. Pan and K. Singleton (2000). Transform analysis and asset pricing for affine jump-diffusions. Econometrica, vol. 68(6):13431376 .

Furrer, H. (1998). Risk processes perturbed by $\alpha$-stable Lévy motion. Scand. Actuar. J., (1):59-74. 
Garrido, J. and M. Morales (2006). On the expected discounted penalty function for Lévy risk processes. N. Am. Actuar. J., vol. 10(4):196-218. Gerber, H. and E. Shiu (1997). The joint distribution of the time of ruin, the surplus immediately before ruin, and the deficit at ruin. Insur. Math. Econ., vol. 21:129-137.

Gerber, H. and E. Shiu (1998). On the time value of ruin. North Am. Actuar. J., vol. 2(1):48-78.

Gerber, H., X. Lin and H. Yang (2006). A note on the dividends-penalty identity and the optimal dividend barrier. ASTIN Bull., vol. 36(2):489503.

Gerber, H. U. and B. Landry (1998). On the discounted penalty at ruin in a jump-diffusion and the perpetual put option. Insur. Math. Econ., vol. 22(3):263-276.

Hubalek, F. and A. Kyprianou (2008). Old and new examples of scale functions for spectrally negative Lévy processes. Tech. rep., http://arxiv.org/abs/0801.0393.

Huzak, M., M. Perman, H. Šikić and Z. Vondraček (2004). Ruin probabilities and decompositions for general perturbed risk processes. Ann. Appl. Probab., vol. 14(3):1378-1397.

Klüppelberg, C. and I. Erder (2008). Quintuple law for sums of dependent Lévy processes. Tech. rep.

Kyprianou, A. (2006). Introductory Lecture Notes on Fluctuations of Lévy Processes with Applications. Springer Verlag.

Kyprianou, A. and R. Loeffen (2008). Refracted Lévy processes. Tech. rep., http://arxiv.org/abs/0801.4655.

Kyprianou, A. and Z. Palmowski (2007). Distributional study of De Finetti's dividend problem for a general Lévy insurance risk process. J. Appl. Probab., vol. 44(2):428-443.

Kyprianou, A. and P. Patie (2008). Transformations of characteristic exponents of convolution semigroups. Tech. rep.

Kyprianou, A. and V. Rivero (2008). Special, conjugate and complete scale functions for spectrally negative Lévy processes. Electron. J. Probab., vol. 13(55):1574-1603.

Kyprianou, A., J. C. Pardo and V. Rivero (2008a). Exact and asymptotic $n$-tuple laws at first and last passage. Tech. rep., http://arxiv.org/abs/0811.3075.

Kyprianou, A., V. Rivero and R. Song (2008b). Convexity and smoothness of scale functions and De Finetti's control problem. Tech. rep., http://arxiv.org/abs/0801.1951.

Kyprianou, A. E. and X. Zhou (2009). General tax structures and the Lévy insurance risk model. Tech. rep., http://arxiv.org/abs/0902.4340. 
Kythe, P. and P. Puri (2002). Computational methods for linear integral equations. Birkhauser.

Lambert, A. (2000). Completely asymmetric Lévy processes confined in a finite interval. Ann. Inst. H. Poincaré Probab. Statist., vol. 36(2):251274 .

Lee, R. (2004). Option pricing by transform methods: Extensions, unification, and error control. J. Comput. Fin., vol. 7(3):51-86.

Lin, X. and G. Willmot (1999). Analysis of a defective renewal equation arising in ruin theory. Ins. Math. Econ., vol. 25(1):63-84.

Loeffen, R. (2008a). An optimal dividends problem with a terminal value for spectrally negative Lévy processes with a completely monotone jump density. Tech. rep., http://www.ricam.oeaw.ac.at/people/page/loeffen/preprints/bankruptcyE1.pdf.

Loeffen, R. (2008b). An optimal dividends problem with transaction costs for spectrally negative Lévy processes. Tech. rep., http://www.ricam.oeaw.ac.at/people/page/loeffen/preprints/impulse_revised12-12-08.pdf.

Loeffen, R. (2009). On optimality of the barrier strategy in de Finetti's dividend problem for spectrally negative Lévy processes. Ann.Appl.Probab., vol. 18(5):1669-1680.

Morales, M. (2007). On the expected discounted penalty function for a perturbed risk process driven by a subordinator. Insur. Math. Econ., vol. 40(2):293-301.

Morales, M. and P. Olivares (2008). On the expected discounted penalty function for risk process driven by a spectrally negative Lévy process. Tech. rep.

Patie, P. (2008). Exponential functional of a new family of Lévy processes and self-similar continuous state branching processes with immigration,. Tech. rep., http://www.staff.unibe.ch/patie/Patie_Exp_CBI.pdf.

Pistorius, M. R. (2005). A potential-theoretical review of some exit problems of spectrally negative Lévy processes. In Séminaire de Probabilités XXXVIII, vol. 1857 of Lecture Notes in Math., pp. 30-41. Springer, Berlin.

Renaud, J.-F. and X. Zhou (2007). Distribution of the present value of dividend payments in a Lévy risk model. J. Appl. Probab., vol. 44(2):420-427.

Rogers, L. (2000). Evaluating first-passage probabilities for spectrally one-sided Lévy processes. J. Appl. Probab., vol. 37:1173-1180.

Surya, B. (2008). Evaluating scale functions of spectrally negative Lévy processes. J. Appl. Probab., vol. 45:135-149. 
Tsai, C. C.-L. and G. E. Willmot (2002). On the moments of the surplus process perturbed by diffusion. Insur. Math. Econ., vol. 31(3):327-350. Yang, H. and L. Zhang (2001). Spectrally negative Lévy processes with applications in risk theory. Adv. Appl. Probab., vol. 33(1):281-291.

Yin, C. and C. Wang (2008). Optimality of the barrier strategy in De Finetti's dividend problem for spectrally negative Lévy processes: An analytical approach. Tech. rep.

Zhou, X. (2005). On a classical risk model with a constant dividend barrier. North Am. Act. J., pp. 95-108. 